\title{
MS03-P11 | A Reliable And Versatile Tool For High-End In-House Structural
}

\section{STUDIES}

Mrosek, Michael (Bruker AXS GmbH, Karlsruhe, GER); Smith, Vernon (Bruker AXS GmbH, Karlsruhe, GER); Adam, Martin (Bruker AXS GmbH, Karlsruhe, GER)

In house X-ray structure determination has seen steady improvements, now being capable of handling ever smaller and weaker diffracting samples. Modern diffraction systems address the need for increased throughput that is essential e.g. for ligand binding studies in the pharmaceutical industry. Performance of these systems has increased so much that protein crystals that previously required synchrotron data collection, now often can be collected in house significantly improving the speed of the projects moving forward.

Here we demonstrate that the D8 VENTURE platform is capable of collecting data of protein crystals with a broad array of challenging properties. High-performance X-ray sources, such as the I $\mathrm{S}$ S DIAMOND, and the PHOTON III-a large active-area photon-counting mixed-mode detector-provide data to high resolution of samples with extremely large unit cells or very small crystal sizes. During development special attention has been given to keeping the system virtually maintenance free and minimizing running costs.

We will also present a number of examples that all benefit from new and advanced system features that were added to achieve an unprecedented ease of use:

- Automated visual and X-ray based crystal centering,

- Improved crystal visualisation features for both, cryo and in situ applications

- Extended import and export functionalities to modern crystallographic pipelines. 